Supplementary Figure 1: Schematic of model progression for vancomycin. Purple square: Final model. OBJ: Objective Function. MDPE: Median Prediction Error. MADPE: Median Absolute Prediction Error. WT: Weight. CPB: Cardiopulmonary Bypass

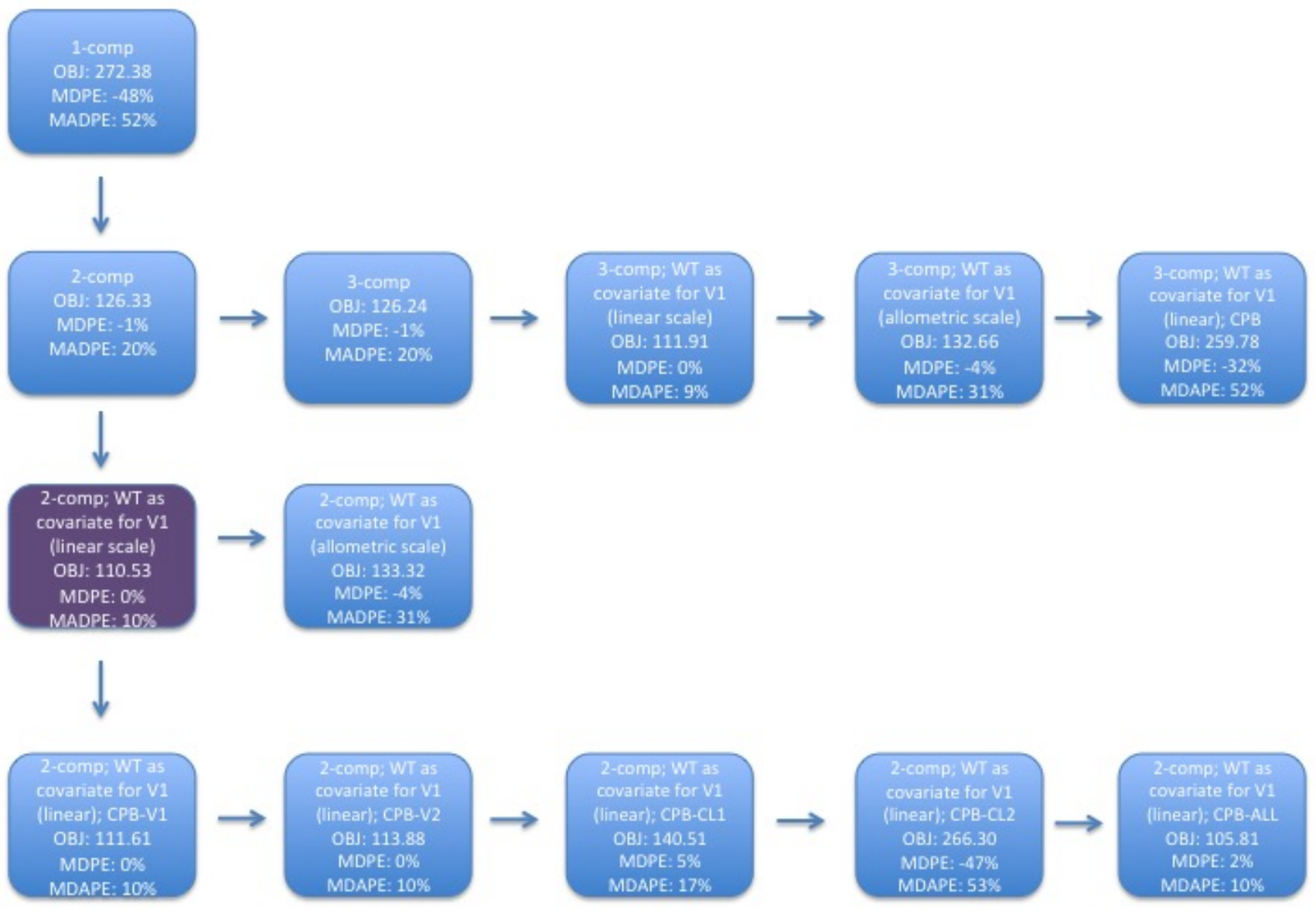


Supplementary Figure 2: Vancomycin Prediction-corrected visual prediction check. Onethousand simulations were performed. $6.4 \%$ of the data fall outside of the $5 \%$ and $95 \%$ confidence intervals.

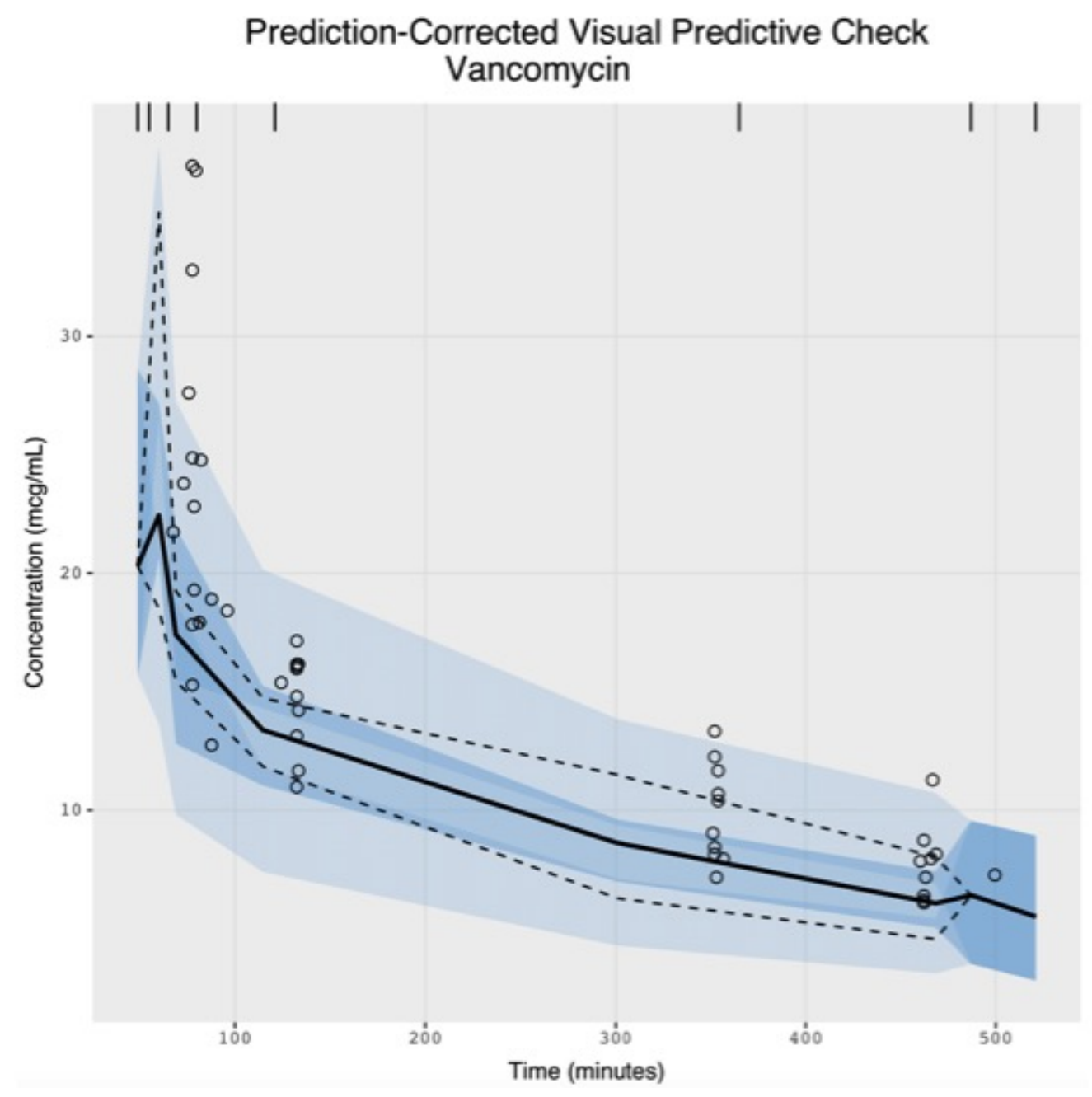


Supplementary Figure 3: Simulation of vancomycin dosing regimen based upon the final model. A dosing regimen of $15 \mathrm{mg} \cdot \mathrm{kg}^{-1}$ administered over 1 hour will maintain vancomycin concentrations above $4 \mu \mathrm{g} \cdot \mathrm{mL}^{-1}$ for 12 hours. Grey circles: Observed concentrations after a single dose. Black line: Infusion duration. Red solid line: Simulated model fit. Red dashed lines: $5 \%$ and $95 \%$ confidence intervals. Black dashed line: Cut-off concentration value (4 $\left.\mu \mathrm{g} \cdot \mathrm{mL}^{-1}\right)$.

Vancomycin Simulation: 1-hour Infusion

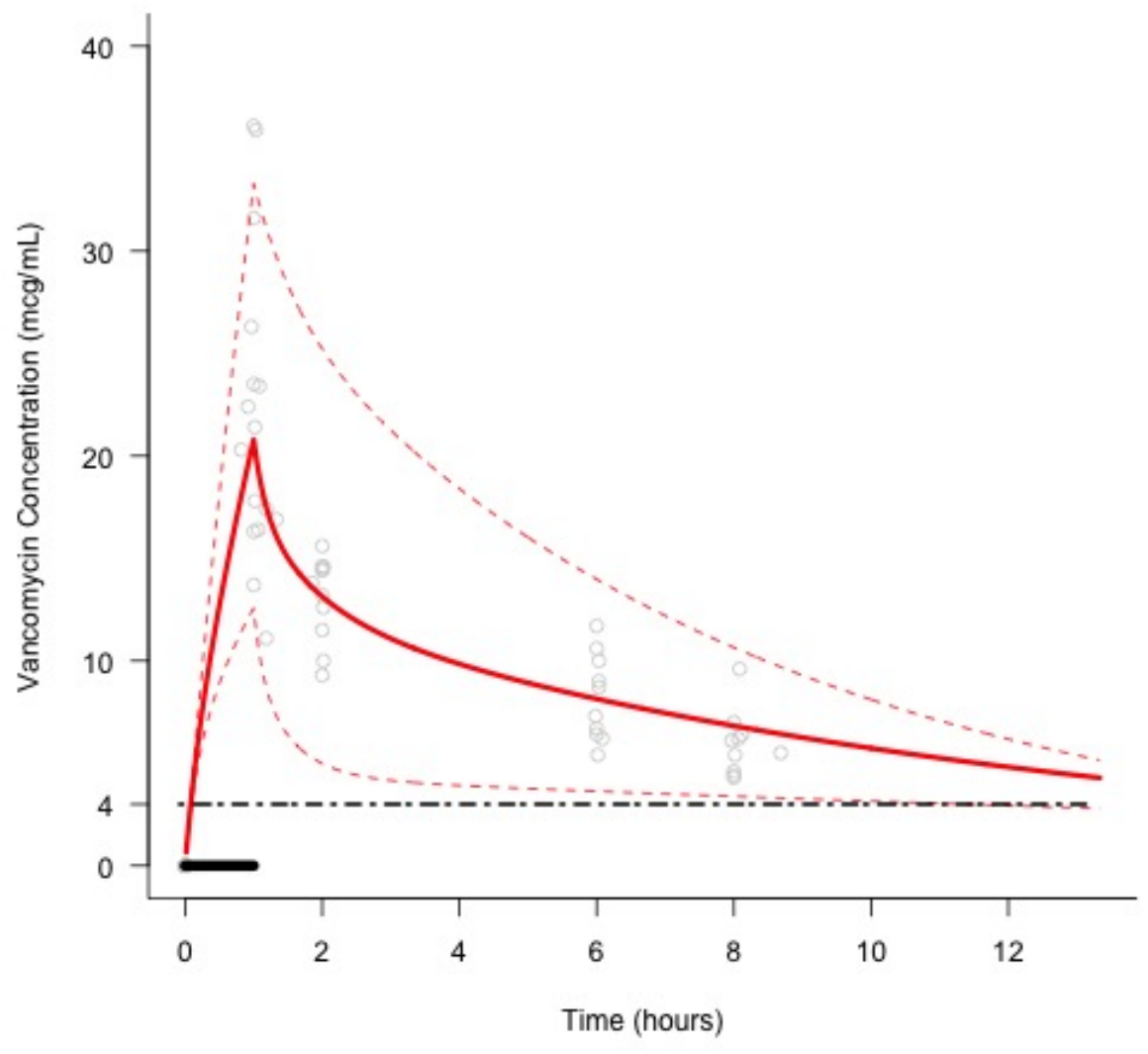


Supplementary Figure 4: Schematic of model progression for cefazolin. Purple square: Final model. OBJ: Objective Function. MDPE: Median Prediction Error. MADPE: Median Absolute Prediction Error. CPB: Cardiopulmonary Bypass

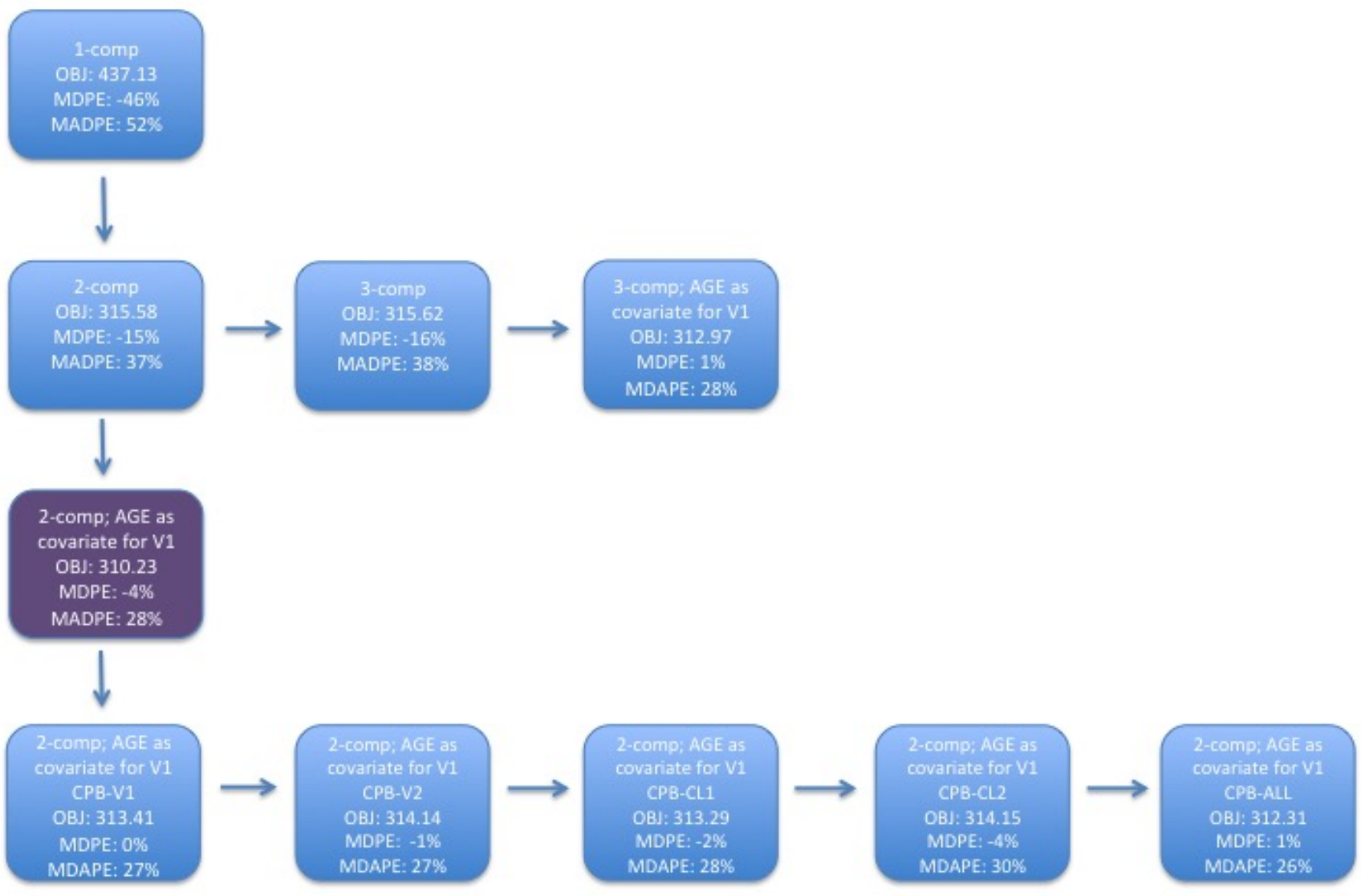


Supplementary Figure 5: Cefazolin Prediction-corrected visual prediction check. Onethousand simulations were performed. $15.7 \%$ of the data fall outside of the $5 \%$ and $95 \%$ confidence intervals.

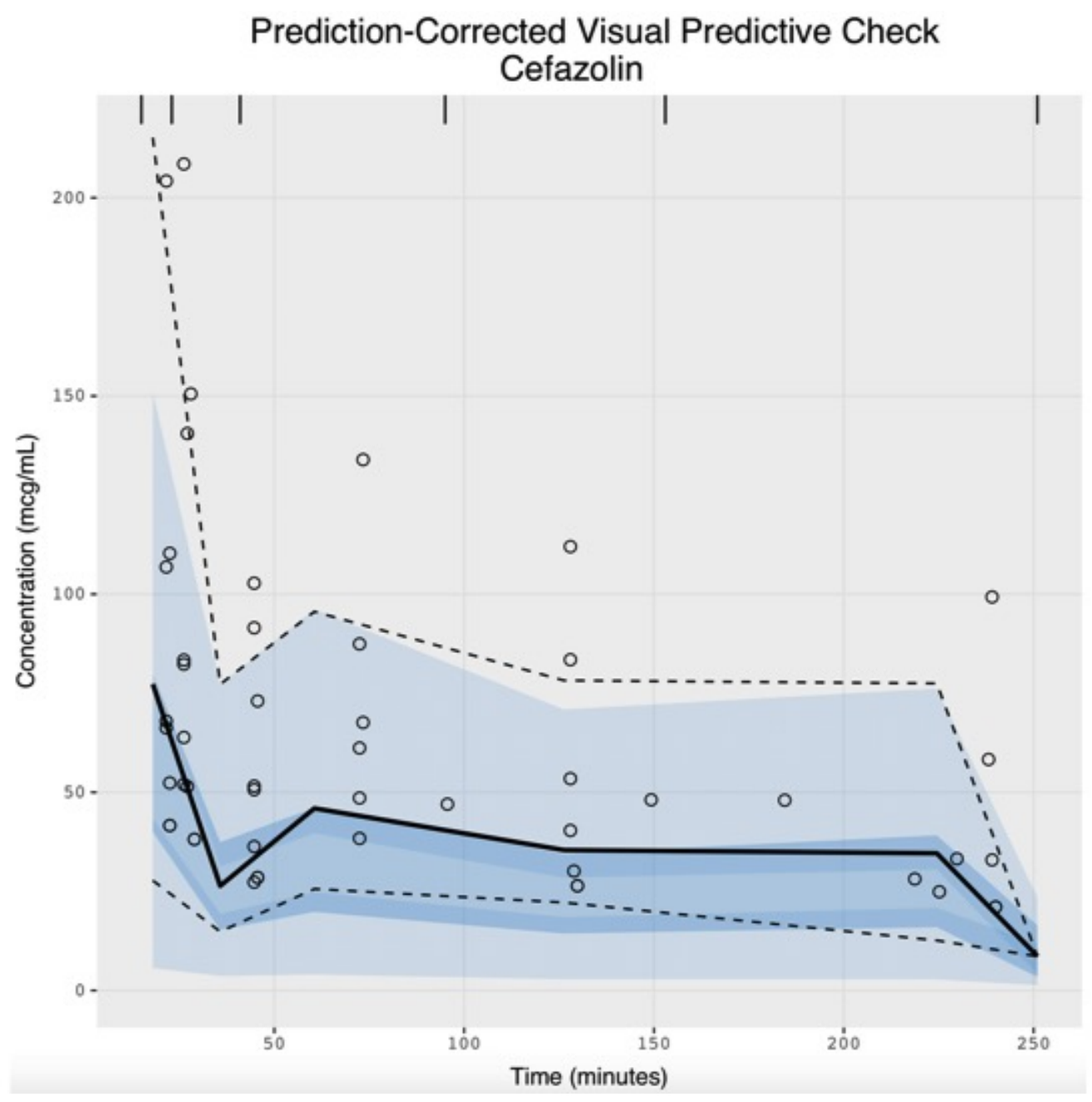


Supplementary Figure 6: Simulation of cefazolin dosing regimen based upon the final model. A dosing regimen of $30 \mathrm{mg} \cdot \mathrm{kg}^{-1} \mathrm{administered} \mathrm{over} 10$ minutes every 4 hours will ensure cefazolin concentrations remain above $16 \mu \mathrm{g} \bullet \mathrm{mL}^{-1}$-the MIC90 for susceptible staphylococci. Grey circles: Observed concentrations after a single dose. Black line: Infusion duration. Red line: Simulated model fit. Red dashed lines: $5 \%$ and $95 \%$ confidence intervals. Black dashed line: Cut-off concentration value $\left(16 \mu \mathrm{g} \cdot \mathrm{mL}^{-1}\right)$.

\section{Cefazolin Simulation}

30 mg/kg; 10-min Infusion; Q4 hour Dosing

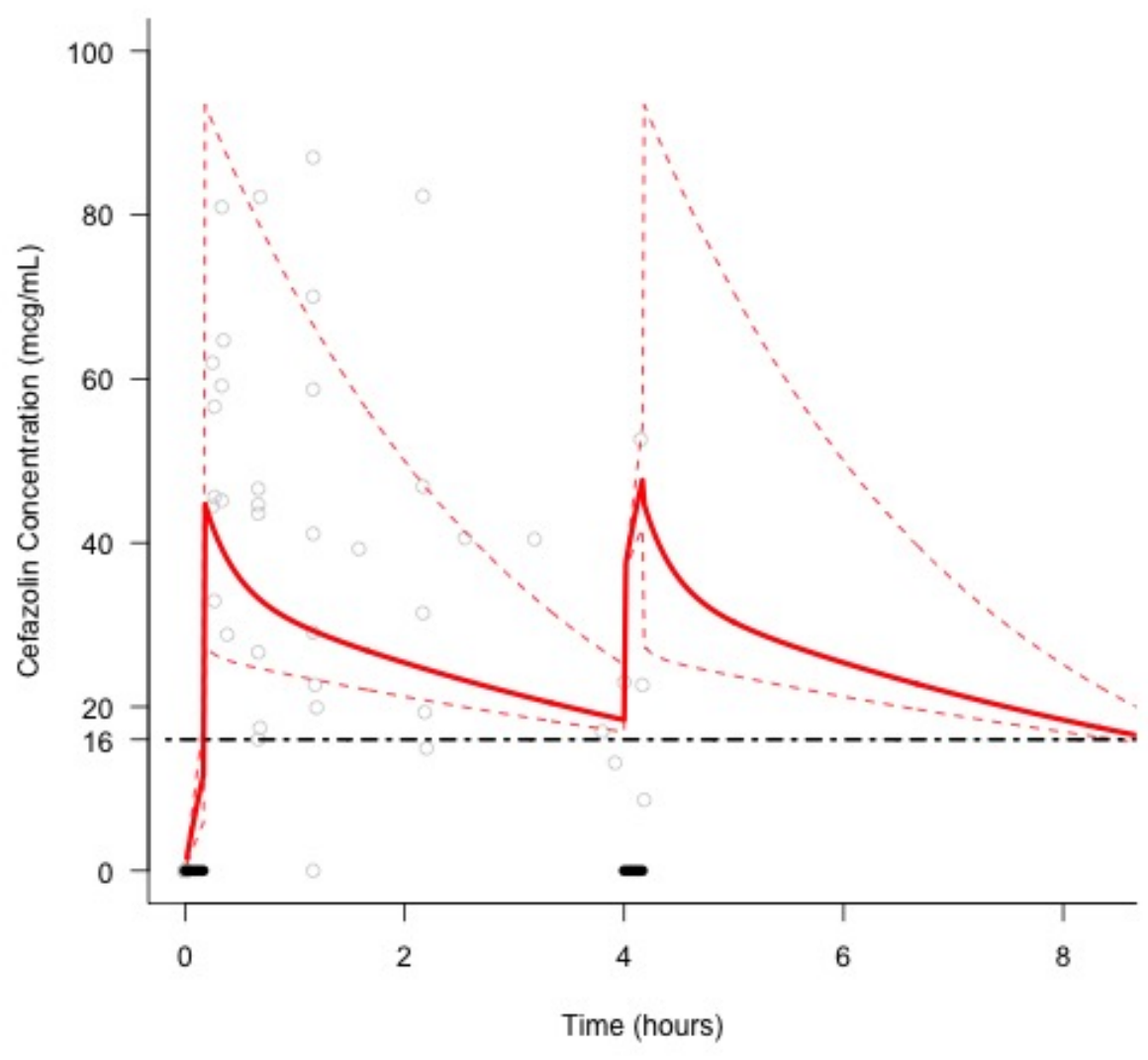

